\title{
EXISTENCE OF STRONG SOLUTIONS OF FULLY NONLINEAR ELLIPTIC EQUATIONS
}

\author{
Adriana Buică \\ Department of Applied Mathematics \\ Babes-Bolyai University of Cluj-Napoca, \\ 1 Kogalniceanu str., RO-3400 Romania \\ abuica@math.ubbcluj.ro
}

\begin{abstract}
The aim of this paper is to study the solvability of the Dirichlet problem for certain types of fully nonlinear elliptic equations. The theory of weakly-near operators, combined to Contraction Mapping and Schauder fixed point theorems, is used. Our main results generalizes similar ones given by S. Campanato and A. Tarsia.
\end{abstract}

Keywords: nonlinear, elliptic, Sobolev spaces, weakly-near operators, fixed point.

\section{Introduction}

In this paper we study the solvability of the Dirichlet problem for certain types of fully nonlinear elliptic equations of the form

$$
u \in H^{2}(\Omega) \cap H_{0}^{1}(\Omega), \quad a\left(x, u, D u, D^{2} u\right)=f(x), \text { for a.e. } x \in \Omega .
$$

In what follows, $\Omega$ will be a $C^{2}$ bounded domain of $\mathbb{R}^{n}$. We denote by $\mathcal{M}_{n}$ the space of $n \times n$ real matrices; $|\cdot|_{m}$ is the euclidean norm in $\mathbb{R}^{m}$; $\operatorname{tr} N=\sum_{i=1}^{n} \xi_{i i}$ is the trace of the $n \times n$ matrix $N=\left(\xi_{i j}\right)$. The Sobolev spaces $H^{2}(\Omega)$ and $H_{0}^{1}(\Omega)$ are as defined in Adams [1975].

We assume that the function $a: \Omega \times \mathbb{R} \times \mathbb{R}^{n} \times \mathcal{M}_{n} \rightarrow \mathbb{R}$ fulfilles the following conditions:

(a1) $a(x, 0,0,0)=0$

(a2) $a(\cdot, r, d, M)$ is measurable,

(a3) $a(x, \cdot, \cdot, \cdot)$ is continuous,

(a4) there exist $\alpha, \beta, \gamma \geq 0$ such that $|a(x, r, d, M)| \leq \alpha|r|+\beta|d|_{n}+\gamma|M|_{n^{2}}$, for all $r \in \mathbb{R}, d \in \mathbb{R}^{n}, M \in \mathcal{M}_{n}$ and for a.e. $x \in \Omega$.

The following ellipticity condition is satisfied.

The original version of this chapter was revised: The copyright line was incorrect. This has been corrected. The Erratum to this chapter is available at DOI: 10.1007/978-0-387-35690-7_44

V. Barbu et al. (eds.), Analysis and Optimization of Differential Systems

(C) IFIP International Federation for Information Processing 2003 
(a5) there exist $c_{1}, c_{2}, c_{3}>0$ with $0<c:=c_{1}-c_{2}-c_{3}<1$ such that $[a(x, r, d, N+M)-a(x, r, d, M)] \operatorname{tr} N \geq c_{1}|\operatorname{tr} N|^{2}-c_{2}|\operatorname{tr} N| \cdot|N|_{n^{2}}-c_{3}|N|_{n^{2}}^{2}$, for all $r \in \mathbb{R}, d \in \mathbb{R}^{n}, M, N \in \mathcal{M}_{n}$ and for a.e. $x \in \Omega$.

We obtain an existence and uniqueness result and another existence result. The theory of weakly-near operators (see Buică and Domokos [2002]), combined to Contraction Mapping Theorem or Schauder Fixed Point Theorem, is used. Our main results are the following.

Theorem 1.1 Let us assume that the function a: $\Omega \times \mathbb{R} \times \mathbb{R}^{n} \times \mathcal{M}_{n} \rightarrow \mathbb{R}$ satisfies (a1)-(a5), and there exist $l_{1}, l_{2}>0$ such that

$$
|a(x, r, d, M)-a(x, s, \delta, M)| \leq l_{1}|r-s|+l_{2}|d-\delta|,
$$

for a.e. $x \in \Omega$ and for all $r, s \in \mathbb{R}, d, \delta \in \mathbb{R}^{n}, M \in \mathcal{M}_{n}$. If $\frac{l_{1}}{\lambda_{1}}+\frac{l_{2}}{\sqrt{\lambda_{1}}}<c$ then (1.1) has a unique solution.

Theorem 1.2 Let us assume that the function a: $\Omega \times \mathbb{R} \times \mathbb{R}^{n} \times \mathcal{M}_{n} \rightarrow \mathbb{R}$ satisfies (a1)-(a5), and there exist $l_{1}, l_{2}>0$ such that

$$
|a(x, r, d, M)-a(x, 0,0, M)| \leq l_{1}|r|+l_{2}|d|,
$$

for a.e. $x \in \Omega$ and for all $r \in \mathbb{R}, d \in \mathbb{R}^{n}, M \in \mathcal{M}_{n}$. If $\frac{l_{1}}{\lambda_{1}}+\frac{l_{2}}{\sqrt{\lambda_{1}}}<c$ then (1.1) has at least one solution.

We extend and generalize similar results of S. Campanato [1989] and A. Tarsia $[1996,1998]$. Another related results are given by D.K. Palagachev [1993], R. Precup [1995, 1997], A. Buică and A. Domokos [2001], A. Buică and F. Aldea [2000], A. Buică [2001b].

The next sections are: 2 . Theoretical preliminaries (necessary results from the theory of weakly-near operators and elliptic equations are presented), 3. Proof of main results and 4. Comments (we explain the relations between our results and those existing in the literature).

\section{Theoretical preliminaries}

Let $X$ be a nonempty set and $Z$ be a Banach space. Let $A, B: X \rightarrow Z$ be two operators. S. Campanato introduced the following notion of nearness between operators in order to use it in the study of fully nonlinear elliptic equations (see Campanato [1989, 1993], Tarsia [1996, 1998]).

Definition 2.1 We say that $A$ is near $B$ if there exists $\alpha>0$ and $0 \leq c<1$ such that

$$
\|B x-B y-\alpha(A x-A y)\| \leq c\|B x-B y\|, \text { for all } x, y \in X .
$$

In a joint paper with $\mathrm{A}$. Domokos, we generalized this notion using an accretivity-type condition, instead of a contraction-type one. 
Let us denote by $\Phi$ the set of all functions $\varphi: \mathbb{R}_{+} \rightarrow \mathbb{R}_{+}$, such that $\varphi(0)=0, \varphi(r)>0$ for $r>0, \liminf _{r \rightarrow \infty} \varphi(r)>0$ and $\liminf _{r \rightarrow r_{0}} \varphi(r)=0$ implies $r_{0}=0$. In this paper we shall refer only to the functions $\varphi$ in $\Phi$. We say that $A$ is $\varphi$-accretive with respect to $B$, if for every $x, y \in X$ there exists $j(B x-B y) \in J(B x-B y)$ such that

$$
\langle A x-A y, j(B x-B y)\rangle \geq \varphi(\|B x-B y\|)\|B x-B y\|,
$$

where $J: Z \leadsto Z^{*}$ is the normalized duality map of $Z$.

The map $A$ is continuous with respect to $B$ if $A \circ B^{-1}: B(X) \sim Z$ has a continuous selection.

The next definition introduce the weak-nearness notion.

Definition 2.2 We say that $A$ is weakly-near $B$ if $A$ is $\varphi$-accretive with respect to $B$ and continuous with respect to $B$.

This notion extends the property of the differential operator to be "near" (or to "approximate") the map, as well as other approximation notions used in nonsmooth theory of inverse or implicit functions (for details in this direction, see Buică and Domokos [2002], Domokos [1997, 2000], Buică [2001b]). The next results will be used in Section 3. They are taken from Buică [2001], Buică and Domokos [2002].

Proposition 2.1 Let $A$ be weakly-near to $B$. If $B$ is bijective, then $A$ is bijective.

Let $z \in Z$ and $A_{1}, A_{2}: X \rightarrow Z$ be two mappings. Let us consider the equation $A_{1}(x)=z$, whose solvability is assured by the weak-nearness between the operator $A_{1}$ and a bijective operator $B: X \rightarrow Z$. Let $x_{1}^{*}$ be a solution of this equation. Let us consider, also, the equation $A_{2}(x)=z$, which is assumed to be solvable. Let $x_{2}^{*}$ be a solution. In the following theorem we shall give an estimation for "the distance" between $x_{1}^{*}$ and $x_{2}^{*}$. This distance depends on the operator $B$.

Theorem 2.1 Let us assume that the following conditions are fullfiled.

(i) $B$ is bijective;

(ii) $A_{1}$ is weakly-near to $B$ with $\varphi(t)=\alpha t, 0<\alpha<1$;

(iii) equation $A_{2}(x)=z$ has at least one solution.

Then we have the estimation $\left\|B\left(x_{1}^{*}\right)-B\left(x_{2}^{*}\right)\right\| \leq \frac{1}{\alpha}\left\|A_{1}\left(x_{2}^{*}\right)-A_{2}\left(x_{2}^{*}\right)\right\|$. If, in addition, there exists $\eta>0$ such that $\left\|A_{1}(x)-A_{2}(x)\right\| \leq \eta$ for all $x \in X$, then $\left\|B\left(x_{1}^{*}\right)-B\left(x_{2}^{*}\right)\right\| \leq \frac{1}{\alpha} \eta$.

We also need the following lemmas which are taken from Gilbarg and Trüdinger [1983], Precup [1997]. 
Lemma 2.1 The Laplace operator $\Delta: H^{2}(\Omega) \cap H_{0}^{1}(\Omega) \rightarrow L^{2}(\Omega)$ is well defined and it is a homeomorphism.

Lemma 2.2 For every $u \in H^{2}(\Omega) \cap H_{0}^{1}(\Omega)$,

$$
\|u\|_{L^{2}} \leq \frac{1}{\lambda_{1}}\|\Delta u\|_{L^{2}}, \quad\|D u\|_{L^{2}} \leq \frac{1}{\sqrt{\lambda_{1}}}\|\Delta u\|_{L^{2}} .
$$

Lemma 2.3 Let $u \in H^{2}(\Omega) \cap H_{0}^{1}(\Omega)$. Then $\left\|D^{2} u\right\|_{2}=\|\Delta u\|_{2}$.

\section{Proof of main results}

Proof of Theorem 1.1. Let $w \in H^{1}(\Omega)$. Let us consider the mapping $A_{w}$ defined by

$$
A_{w}: H^{2}(\Omega) \cap H_{0}^{1}(\Omega) \rightarrow L^{2}(\Omega), \quad A_{w}(u)(x)=a\left(x, w, D w, D^{2} u\right) .
$$

Let us consider, also, the equation

$$
A_{w}(u)=f
$$

1) $A_{w}$ is well defined and continuous.

Using condition (a4) we obtain that

$$
\left|a\left(x, u, D u, D^{2} u\right)\right| \leq \alpha|u(x)|+\beta|D u(x)|_{n}+\gamma\left|D^{2} u\right|_{n^{2}},
$$

for every $u \in H^{2}(\Omega) \cap H_{0}^{1}(\Omega)$.

Because the right hand side of this inequality is an $L^{p}$-function, we can deduce that $A$ is well-defined and continuous.

2) $A_{w}$ is weakly near to $\Delta$.

The mapping $A_{w}$ is continuous with respect to $B=\Delta$ because $A_{w}$ and $B^{-1}$ are continuous. We shall prove that $A_{w}$ is strongly accretive (in fact, strongly monotone) with respect to $B$. Using (a5) and Lemma 2.3 we obtain:

$$
\begin{aligned}
& \left\langle A_{w} u-A_{w} v, \Delta u-\Delta v\right\rangle_{L^{2}}= \\
& =\int_{\Omega}\left[a\left(x, w, D^{2} u\right)-a\left(x, w, D^{2} v\right)\right] \cdot \Delta(u-v) d x \geq \\
& \geq \int_{\Omega} c_{1}|\Delta(u-v)|^{2}-c_{2}|\Delta(u-v)| \cdot\left|D^{2}(u-v)\right|-c_{3}\left|D^{2}(u-v)\right|^{2} d x \geq \\
& \geq\left(c_{1}-c_{2}-c_{3}\right)\|\Delta(u-v)\|_{L^{2}}^{2} .
\end{aligned}
$$

Thus

$$
\left\langle A_{w}(u)-A_{w}(v), \Delta u-\Delta v\right\rangle_{L^{2}} \geq c\|\Delta u-\Delta v\|_{L^{2}}^{2} .
$$

$A_{w}$ is weakly near to $B$, which is a bijective map. Then, using Proposition 2.1, $A_{w}$ is bijective. Thus, equation (3.1) has a unique solution, let us denote it by $u_{w}$. 
Let us consider now another operator, related to equation (3.1), defined by

$$
\mathcal{U}: H^{1}(\Omega) \rightarrow L^{2}(\Omega), \quad \mathcal{U}(w)=-\Delta u_{w} .
$$

Let us notice that $v=-\Delta w$ is a fixed point of

$$
\mathcal{U} \circ(-\Delta)^{-1}: L^{2}(\Omega) \rightarrow L^{2}(\Omega)
$$

if and only if $u_{w}=w$, which means that $u_{w}$ is a solution of the problem (1.1).

Let $w_{1}, w_{2} \in H^{1}(\Omega)$ and let us consider the mappings $A_{w_{1}}$ and $A_{w_{2}}$. For every $u \in H^{2}(\Omega) \cap H_{0}^{1}(\Omega)$, using relation (1.2) and Lemma 2.2, we obtain the estimations,

$$
\begin{aligned}
& \left\|A_{w_{1}}(u)-A_{w_{2}}(u)\right\|_{L^{2}}= \\
& =\left\|a\left(\cdot, w_{1}(\cdot), D w_{1}(\cdot), D^{2} u(\cdot)\right)-a\left(\cdot, w_{2}(\cdot), D w_{2}(\cdot), D^{2} u(\cdot)\right)\right\|_{L^{2}} \leq \\
& \leq\left\|l_{1}\left|w_{1}(\cdot)-w_{2}(\cdot)\right|+l_{2} \mid D w_{1}(\cdot)-D w_{2}(\cdot)\right\|_{L^{2}} \leq \\
& \leq l_{1}\left\|w_{1}-w_{2}\right\|_{L^{2}}+l_{2}\left\|D w_{1}-D w_{2}\right\|_{L^{2}} \leq \\
& \leq\left(\frac{l_{1}}{\lambda_{1}}+\frac{l_{2}}{\sqrt{\lambda_{1}}}\right)\left\|\Delta w_{1}-\Delta w_{2}\right\|_{L^{2}}:=\eta .
\end{aligned}
$$

We apply Theorem 2.1 and obtain $\left\|B\left(u_{w_{1}}\right)-B\left(u_{w_{2}}\right)\right\|_{L^{2}} \leq \frac{1}{c} \eta$, which means that

$$
\left\|\mathcal{U}\left(w_{1}\right)-\mathcal{U}\left(w_{2}\right)\right\|_{L^{2}} \leq \frac{1}{c}\left(\frac{l_{1}}{\lambda_{1}}+\frac{l_{2}}{\sqrt{\lambda_{1}}}\right) \cdot\left\|\Delta w_{1}-\Delta w_{2}\right\|_{2} .
$$

Then $\mathcal{U} \circ(-\Delta)^{-1}$ is a contraction on $L^{2}(\Omega)$. By Contraction Mapping Theorem, it has a unique fixed point, $v^{*}$. If we denote by $w^{*}=$ $(-\Delta)^{-1} v^{*}$, then $u_{w^{*}}$ is the unique solution of (1.1).

Proof of Theorem 1.2. It is possible to consider again the mapping $\mathcal{U}$ like in the proof of Theorem 1.1. Let us notice that $w$ is a fixed point of

$$
(-\Delta)^{-1} \circ \mathcal{U}: H^{1}(\Omega) \rightarrow H^{1}(\Omega)
$$

if and only if $u_{w}$ is a solution of the problem (1.1). This time we shall apply the Schauder Fixed Point Theorem.

First we shall prove that $\mathcal{U}$ is continuous in every $v \in H^{1}(\Omega)$. Let us denote by $u_{v} \in H^{2}(\Omega) \cap H_{0}^{1}(\Omega)$ the unique solution of $A_{v}(u)=f$, with $A_{v}$ defined like in the proof of previous theorem. The hypotheses (a3) and (a4) assure that the mapping $w \longmapsto a\left(\cdot, w, D w, D^{2} u_{v}\right)$ is continuous from $H^{1}(\Omega)$ to $L^{2}(\Omega)$. In particular, is continuous in $v$. Then, for every $\varepsilon>0$ there exists $\delta>0$ such that, whenever $w \in H^{1}(\Omega),\|w-v\|_{H^{1}}<\delta$,

$$
\left\|a\left(\cdot, w, D w, D^{2} u_{v}\right)-a\left(\cdot, v, D v, D^{2} u_{v}\right)\right\|_{L^{2}}<\varepsilon .
$$


Then, $\left\|A_{w}\left(u_{v}\right)-A_{v}\left(u_{v}\right)\right\|_{L^{2}} \leq \varepsilon$. We apply Theorem 2.1 like in the proof of the previous theorem and obtain $\|\mathcal{U}(w)-\mathcal{U}(v)\|_{L^{2}} \leq \frac{1}{c} \cdot \varepsilon$. Hence, $\mathcal{U}$ is continuous, indeed.

Let us consider now the following norm in $H^{1}(\Omega)$,

$$
\|w\|_{*}=l_{1}\|w\|_{L^{2}}+l_{2}\|D w\|_{L^{2}}
$$

which is equivalent to the usual norm,

$$
\|w\|_{H^{1}}=\left(\int_{\Omega}|w|^{2}+|D w|^{2} d x\right)^{1 / 2} .
$$

Let $w \in H^{1}(\Omega)$ and let us consider the mappings $A_{w}$ and $A_{0}$. For every $u \in H^{2}(\Omega) \cap H_{0}^{1}(\Omega)$ we obtain the following estimations like in the proof of the previous theorem,

$$
\left\|A_{w}(u)-A_{0}(u)\right\|_{L^{2}} \leq l_{1}\|w\|_{L^{2}}+l_{2}\|D w\|_{L^{2}}=\|w\|_{*} .
$$

We apply Theorem 2.1 and obtain $\|\mathcal{U}(w)-\mathcal{U}(0)\|_{L^{2}} \leq \frac{1}{c}\|w\|_{*}$. Then

$$
\|\mathcal{U}(w)\|_{L^{2}} \leq \frac{1}{c}\|w\|_{*}+\|\mathcal{U}(0)\|_{L^{2}}
$$

This assures that $\mathcal{U}:\left(H^{1}(\Omega),\|\cdot\|_{*}\right) \rightarrow\left(L^{2}(\Omega),\|\cdot\|_{L^{2}}\right)$ is a bounded operator. Also, we have that $(-\Delta)^{-1}: L^{2}(\Omega) \rightarrow H^{2}(\Omega) \cap H_{0}^{1}(\Omega)$ is bounded and $H^{2}(\Omega)$ is compactly imbedded in $H^{1}(\Omega)$. Hence, $(-\Delta)^{-1} \circ \mathcal{U}: H^{1}(\Omega) \rightarrow H^{1}(\Omega)$ is completely continuous.

Now we prove that there exists an invariant set of $(-\Delta)^{-1} \circ \mathcal{U}$. The following relations hold for every $w \in H^{1}(\Omega)$,

$$
\left\|(-\Delta)^{-1} \circ \mathcal{U}(w)\right\|_{*} \leq\left(\frac{l_{1}}{\lambda_{1}}+\frac{l_{2}}{\sqrt{\lambda_{1}}}\right)\|\mathcal{U}(w)\|_{L^{2}} \leq l_{3}\|w\|_{*}+c l_{3}\|\mathcal{U}(0)\|_{L^{2}}
$$

where $l_{3}:=\frac{1}{c}\left(\frac{l_{1}}{\lambda_{1}}+\frac{l_{2}}{\sqrt{\lambda_{1}}}\right)$. We have used the definitions of $\mathcal{U}$ and $\|w\|_{*}$, Lemma 2.2 and relation (3.3). Since $l_{3}<1$, we let

$$
R \geq \frac{c l_{3}\|\mathcal{U}(0)\|_{L^{2}}}{1-l_{3}}
$$

We have that $\left\|(-\Delta)^{-1} \circ \mathcal{U}(w)\right\|_{*} \leq R$ whenever $\|w\|_{*} \leq R$, i.e. the ball centered in origin with radius $R$ from the Banach space $\left(H^{1}(\Omega),\|\cdot\|_{*}\right)$, is an invariant set for the mapping $(-\Delta)^{-1} \circ \mathcal{U}$.

Applying the Schauder fixed point theorem we deduce that $(-\Delta)^{-1} \circ \mathcal{U}$ has at last one fixed point, $w^{*}$. Then $u_{w^{*}}$ is a solution of (1.1). 


\section{Comments}

In this section we relate our results to some other ones in the literature. S. Campanato [1989] and A. Tarsia [1996, 1998] considered a function $a=a(x, M)$ (i.e. the equation (1.1) does not depend explicitely on the function $u$ and its gradient) which satisfies (a1)-(a3) and the following ellipticity condition:

(a6) there exist three positive constants $\alpha, \beta, \gamma$, with $\gamma+\delta<1$ such that

$$
|\operatorname{tr} N-\alpha[a(x, M+N)-a(x, M)]| \leq \gamma|N|_{n^{2}}+\delta|\operatorname{tr} N|,
$$

for almost every $x \in \Omega$, for all $M, N \in \mathcal{M}_{n}$.

This is stronger than (a5)+(a4). Hence, the particular case of Theorem 1.1 when $a=a(x, M)$ generalizes the main result in Campanato [1989].

In Tarsia [1998] the following problem is also considered

$$
u \in H^{2}(\Omega) \cap H_{0}^{1}(\Omega), a\left(x, D^{2} u\right)+g(x, u)=f(x), \text { for a.e. } x \in \Omega \text {. }
$$

The function $g: \Omega \times \mathbb{R} \rightarrow \mathbb{R}$ is measurable and there exists $l \leq \lambda_{1}$ (where $\lambda_{1}$ is the smallest positive eigenvalue of the operator $-\Delta$ ), and for all $r, s \in \mathbb{R}$ and for a.e. $x \in \Omega$

(i) $g(x, 0)=0$,

(ii) $0 \leq[g(x, r)-g(x, s)](r-s)$,

(iii) $|g(x, r)-g(x, s)| \leq l|r-s|$.

In Tarsia [1998] an existence and uniqueness result is obtained for (4.4) when $l$ is sufficiently small. It is easily seen that this result is also a consequence of Theorem 1.1.

Campanato [1989] and Tarsia [1998] used the theory of near-operators. The theory of weakly-near operators permited us to deal with the weaker ellipticity condition (a5). D. Palagachev [1993] studied the solvability of the Dirichlet problem for a class of fully nonlinear elliptic equations under ellipticity condition (a6). The equation contained a term of the form $f=f(x, u, D u)$. The author combined the theory of near-operators to the Leray-Schauder Fixed Point Theorem. We gave some existence and uniqueness results in $W^{2, p}(\Omega) \cap W_{0}^{1}(\Omega)$ (with an arbitrary $p>1$ ) for equation $a\left(x, u, D u, D^{2} u\right)=f$ (see Buică and Domokos [2002]). We used another ellipticity condition which assured the weak nearness to a general linear elliptic operator. The results of this paper extends similar ones appeared in Buică [2001b], where the gradient did not appear in the form of the equation. Our joint paper, Buică and Aldea [2000], contains a data dependence theorem for equations of the form $a\left(x, D^{2} u\right)=f$ in $H^{2}(\Omega) \cap H_{0}^{1}(\Omega)$. Also these results extends to the case of fully nonlinear equations those given by $R$. Precup [1995, 1997] for semilinear elliptic equations. 


\section{References}

[1] R. A. Adams, Sobolev Spaces, Academic Press, New York/San Francisco/London, 1975.

[2] A. Buică and F. Aldea, On Peetre's condition in the coincidence theory, Proc. of Séminaire de la theorie de la meilleure approximation, Cluj-Napoca, pg. 17-27, october 2000.

[3] A. Buică, Some properties preserved by weak nearness, Seminar on fixed point theory Cluj-Napoca 2: 65-71, 2001.

[4] A. Buică, Principii de coincidenţă şi aplicaţii (Coincidence Principles and Applications, in romanian), Cluj University Press, Cluj-Napoca, 2001.

[5] A. Buică and A. Domokos, Nearness, accretivity and the solvability of nonlinear equations, Numer. Funct. Anal. Appl. 23:477-493, 2002.

[6] S. Campanato, A Cordes type condition for nonlinear variational systems, Rend. Acc. Naz. delle Sc. 107: 307-321, 1989.

[7] S. Campanato, Further contribution to the theory of near mappings, Le Matematiche 48: 183-187, 1993.

[8] A. Domokos, Teoreme de functiii implicite nenetede şi aplicaţiile lor (Nonsmooth implicit functions and their applications, in romanian), Babeş-Bolyai University, Cluj-Napoca, Doctoral thesis, 1997.

[9] A. Domokos, Implicit function theorems for m-accretive and locally accretive set-valued mappings, Nonlinear Analysis 41: 221-241, 2000.

[10] D. Gilbarg and N.S. Trudinger, Elliptic Partial Differential Equations of Second Order, Springer Verlag, 1983.

[11] D.K. Palagachev, Global strong solvability of Dirichlet problem for a class of nonlinear elliptic equations in the plane, Le Matematiche 48: 311-321, 1993.

[12] R. Precup, Existence results for nonlinear boundary value problems under nonresonance conditions, Qualitative Problems for Differential Equations and Control Theory (C. Corduneanu, ed.) World Scientific, Singapore, pp. 263-273, 1995.

[13] R. Precup, Ecuatii cu Derivate Partiale, Transilvania Press, Cluj, 1997.

[14] A. Tarsia, Some topological properties preserved by nearness between operators and applications to PDE, Czech. Math. J. 46(121): 607-624, 1996.

[15] A. Tarsia, Differential equations and implicit function: a generalization of the near operators theorem, Topol. Meth. Nonlin. Anal. 11: 115-133, 1998. 